\title{
A NOTE ON THE GLOBAL DIMENSION OF SUBRINGS
}

\section{F. L. SANDOMIERSKI ${ }^{1}$}

1. Introduction. Let $R$ be a ring with identity and $e$ an idempotent of $R$. Chase [3] shows that if each principal right ideal of $R$ is $R$-projective ( $R$ is a right PP ring), then $e R e$ is a right PP ring. Small [6], shows that if $R$ is right Noetherian and right hereditary, then so is $e R e$.

In this note it is shown that if $R$ satisfies one of the properties, right $\mathrm{PP}$, right semihereditary, right hereditary or $\mathrm{WGD}(R) \leqq 1$, then $e R e$ satisfies the same property. An example is given for which the right global dimension of $R, r G D(R)$, is 2 , but $r G D(e R e)$ is infinite.

2. Definitions and notation. $R$ always denotes a ring with identity and all modules are unital. The Jacobson radical of $R$ is denoted $J=J(R)$. A ring $R$ is local if $J$ consists of all the nonunits.

If $e$ is an idempotent of $R$, then a right $R$-module $P_{R}$ is $e$-free, if it is a direct sum of copies of $e R$. A right $R$-module is $e$-accessible if it is the epimorphic image of an $e$-free module.

For homological notions the reader is referred to [1].

3. Results. In the following $\otimes$ denotes tensor product over $R$.

Theorem 1. Let $R$ be a ring and $e$ an idempotent of $R, S=e R e$, and $M_{R}$ an e-accessible $R$-module such that $M$ is $R$-projective ( $R$-flat), then $(M \otimes R e)_{S}$ is $S$-projective ( $S$-flat).

Proof. By hypothesis there is an exact sequence of right $R$-modules

$$
0 \rightarrow K \rightarrow P \rightarrow M \rightarrow 0
$$

with $P e$-free, hence projective. Since $R e$ is a flat left $R$-module the induced sequence of right $S$-modules

$$
0 \rightarrow K \otimes \operatorname{Re} \rightarrow P \otimes \operatorname{Re} \rightarrow M \otimes \operatorname{Re} \rightarrow 0
$$

is exact.

If $M$ is $R$-projective, then (1) is split exact and therefore so is (2). Since $P \otimes R e$ is a free $S$-module it follows that $M \otimes R e$ is $S$-projective.

Now suppose that $M$ is $R$-flat. First, we remark that [2, Proposition 2.2] is valid if $F_{R}$ is $R$-projective and not necessarily $R$-free as may be easily verified. In view of this remark it is sufficient to show that for $v \in K \otimes R e$, there is an $S$-homomorphism $\phi: P \otimes R e \rightarrow K \otimes R e$

\footnotetext{
1 This paper was written with partial support from NSF Grant GP-7073.
} 
such that $\phi(v)=v$. Clearly, $v=u \otimes e$ for some $u$ in $K$, so there is an $R$-homomorphism $\psi: P \rightarrow K$ such that $\psi(u)=u$, then the desired map is $\phi=\psi \otimes 1$ and the theorem follows.

Corollary 1. If $R$ is a ring, $e$ an idempotent of $R, S=e R e$, and $R$ satisfies one of the properties, right $P P$, right semihereditary, right hereditary or $\mathrm{WGD}(R) \leqq 1$, then $S$ satisfies the same property.

Proof. Let $I$ be a right ideal of $S$, and $I R$ the right ideal of $R$ generated by $I$, then clearly $I R$ is $e$-accessible and finitely generated on the same number of generators as $I$, if $I$ is finitely generated. Now, $I R \otimes R e$ is $S$-isomorphic to $I R R e=I e R e=I$ and the corollary follows.

CoROllaRy 2. Let $P$ be a finitely generated projective right $R$-module and $S$ its $R$-endomorphism ring. If $R$ is right semihereditary, right hereditary or $\mathrm{WGD}(R) \leqq 1$, then $S$ has the same property.

Proof. $P$ is a direct summand of a free right $R$-module $F$ with a finite basis, say $n$ elements. By writing $R$-endomorphisms of $F$ to the left of elements of $F$, the $R$-endomorphism ring of $F$ may be identified with $R_{n}$, the ring of all $n \times n$ matrices over $R$. If $R$ has one of the properties mentioned in the hypothesis of this corollary, then $R_{n}$ has the same property by [5], or what is the same, by Morita equivalence of the categories of right $R$-modules and right $R_{n}$-modules.

Since $P$ is a direct summand of $F, P=e F$ for some idempotent $e$ of $R_{n}$ and therefore $S$ is ring isomorphic to $e R_{n} e$ and this corollary follows from Corollary 1.

An example will show that there is no general theorem: If $\operatorname{rGD}(R)$ $\leqq n$, then $\operatorname{rGD}(e R e) \leqq n$; for $n \geqq 2$. The difficulty arises in the fact that submodules of $e$-free modules are not necessarily $e$-accessible, otherwise the obvious techniques would yield such a theorem.

The following example is from [4]. Let $K$ be a field and $R$ the ring of all $3 \times 3$ matrices of the form

$$
\left[\begin{array}{lll}
a & 0 & 0 \\
c & b & 0 \\
d & f & a
\end{array}\right], \quad \text { where } a, b, c, d, f \text { are in } K .
$$

In [4] it is shown, or it may be easily verified that $R$ is a semiprimary ring with $\operatorname{rGD}(R)=1 \mathrm{GD}(R)=2$. Let $e$ be the idempotent

$$
e=\left[\begin{array}{lll}
1 & 0 & 0 \\
0 & 0 & 0 \\
0 & 0 & 1
\end{array}\right]
$$


then $e R e$ is the ring of all matrices of the form

$$
\left[\begin{array}{lll}
a & 0 & 0 \\
0 & 0 & 0 \\
f & 0 & a
\end{array}\right], \quad \text { where } a, f \text { are in } K .
$$

It is easily verified that $e R e$ is a local ring whose Jacobson radical is a simple left and right ideal of $e R e$, which is isomorphic to the ring modulo its Jacobson radical necessarily, hence is not $e R e$ projective. This fact yields a projective resolution of this simple ideal $U$ of the form (regarded as a left or right $e R e$ module)

$$
\cdots e R e \rightarrow e R e \rightarrow e R e \rightarrow U \rightarrow 0,
$$

so $\mathrm{rGD}(e R e)=1 \mathrm{GD}(e R e)$ is infinite.

\section{REFERENCES}

1. H. Cartan and S. Eilenberg, Homological algebra, Princeton Univ. Press, Princeton, N. J., 1956.

2. S. U. Chase, Direct products of modules, Trans. Amer. Math. Soc. 97 (1960), $457-473$.

3. - A Generalization of the ring of triangular matrices, Nagoya Math. J. 18 (1961), 13-25.

4. S. Eilenberg, H. Nagao and T. Nakayama, On the dimension of modules and algebras. IV, Nagoya Math. J. 10 (1956), 87-95.

5. M. Harada, Note on the dimension of modules and algebras, J. Inst. Polytech. Osaka City Univ. Ser. A 7 (1956), 17-27.

6. L. W. Small, Semihereditary rings, Bull. Amer. Math. Soc. 73 (1967), 656-658.

UNIVERSity OF Wisconsin, Madison 